\title{
A rare but serious complication of GreenLight HPS photoselective vaporization of the prostate: Prostatic capsular perforation with bilateral thigh urinomas and osteitis pubis
}

\author{
David Harriman, MSI; Brian E. Mayson, MD; Ercole F. Leone, MD, FRCSC
}

University of British Columbia (UBC) Faculty of Medicine and UBC Department of Urological Sciences, Vancouver, BC

Cite as: Can Urol Assoc J 2013;7:E105-E107; http://dx.doi.org/10.5489/cuai.230

\section{Abstract}

The use of lasers to perform photoselective vaporization of the prostate (PVP) has been widely accepted as a safe and effective treatment for benign prostatic hyperplasia with very few reported complications. To date, most of the published data report outcomes for the 80-W potassium-titanyl-phosphate laser. A more potent laser, the 120-W GreenLight HPS, was introduced in 2006 and provides more efficient vaporization of prostatic tissue and decreased operating times. Despite these benefits, the increased energy applied to the prostate evokes concerns of potential serious complications, including capsular perforation and injury to adjacent structures. A more powerful laser system, the 180-W GreenLight XPS laser (American Medical Systems, Minnetonka, $\mathrm{MN}$ ) has recently become available. We report a rare but serious complication of GreenLight HPS PVP resulting in prostatic capsular perforation with urinary extravasation, presenting with bilateral thigh urinomas and osteitis pubis.

\section{Case report}

A previously healthy 68-year-old man presented for treatment of his symptomatic benign prostatic hyperplasia (BPH). Initially he had bothersome obstructive urinary symptoms, an International Prostate Symptom Score (IPSS) of 22 (severely symptomatic), a postvoid residual (PVR) volume of 400 to $500 \mathrm{cc}$ and a prostate size of $38 \mathrm{cc}$. He was started on an alpha-blocker, given clean intermittent catheterization $(\mathrm{CIC})$ and counselled about surgical options for management.

He chose to have photoselective vaporization of the prostate (PVP) using the 120-W GreenLight HPS laser (American Medical Systems, Minnetonka, MN), a treatment option not currently offered at our local hospitals. Unfortunately, the complete details of the surgical procedure are not known as it was performed at an outside institution in another country. Preoperative urine culture was reportedly negative, perioperative antibiotics were administered and, to the best of the patient's knowledge, the procedure was free of complications. On postoperative day 2, his Foley catheter was removed and he was discharged with no scheduled follow-up.

Within hours after discharge, the patient was unable to void so he decided to re-initiate CIC on his own accord. Upon returning home on postoperative day 4, he presented to a local emergency department with ongoing retention and dysuria. A computed tomography (CT) scan revealed inflammatory changes suspicious for infection, however urine culture failed to grow an offending organism. An indwelling Foley catheter was placed and antibiotics were initiated. Ten days later, after confirming sterile urine cultures, the Foley catheter was removed and the antibiotics were discontinued. Two weeks postoperatively he was voiding volitionally with mild stress incontinence and a PVR of $30 \mathrm{cc}$.

One month postoperatively, he presented to our emergency department with bilateral thigh swelling, an increased white blood cell count $\left(12.9 \times 10^{9} / \mathrm{L}\right)$, fever $\left(38.2^{\circ} \mathrm{C}\right)$ and severe pubic pain that limited his mobility. His urine culture was positive for multi-drug resistant E. coli and ultrasoundguided aspiration of the thigh collections was consistent with infected urinomas.

His CT cystogram (Fig. 1) and voiding cystourethrogram (Fig. 2) demonstrated extravasation of urine through the anterior prostatic capsule that traversed the pubic symphysis into the medial aspects of the right and left adductor muscles. Cystoscopic images demonstrated a large anterior perforation of the capsule surrounded by necrotic prostatic tissue and magnetic resonance imaging showed evidence of osteitis pubis. 


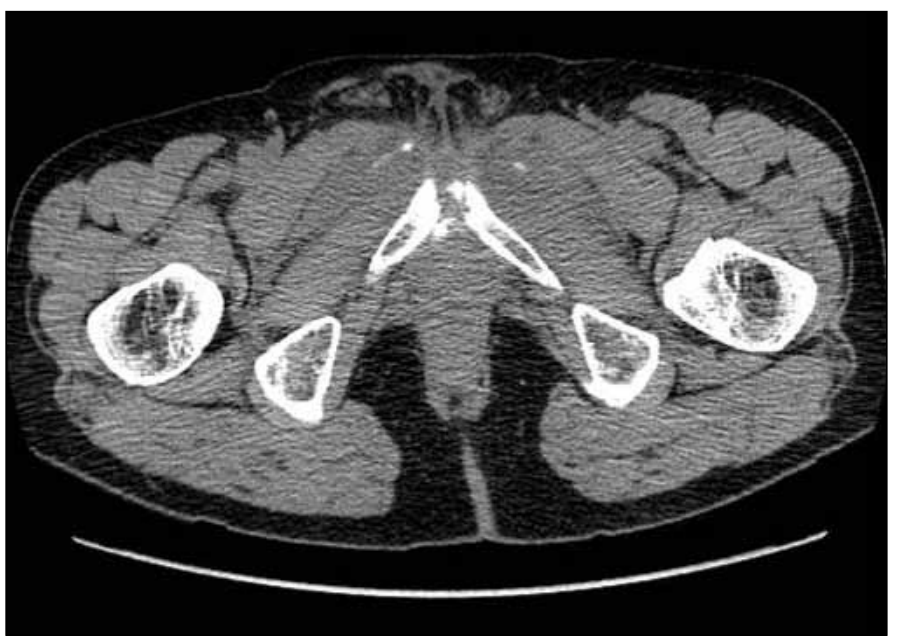

Fig. 1. A computed tomography cystogram, demonstrating bilateral fluid collections in the medial thigh, revealed to be infected urinomas.

Following 6 weeks of broad-spectrum antibiotic treatment and 4 months of Foley catheterization with suprapubic urinary diversion, follow-up imaging demonstrated a persistent anterior prostatic capsule urine leak.

After failure of conservative management, the patient went on to have open operative fistula repair with a peritoneal interposition flap. Visualization of the prostate during the procedure revealed an atrophic appearing gland with a 15-mm long anterior capsular defect surrounded by necrotic, distorted tissue and in direct communication with an area of the pubic symphysis devoid of periosteum. Post-repair imaging confirmed closure of the fistulous tract with no ongoing extravasation of urine. The pelvic pain is resolving, however, the patient continues to suffer from stress urinary incontinence, mild erectile dysfunction that responds to phosphodiesterase type 5 inhibitor therapy and anejaculation that is new since the PVP procedure.

\section{Discussion}

PVP is widely accepted as a safe and effective alternative to traditional transurethral resection of the prostate (TURP) for the endoscopic treatment of $\mathrm{BPH}$. Most of the published data for PVP include outcomes for the 80-W potassiumtitanyl-phosphate (KTP) laser; this laser was first introduced in the early 2000s and uses the green spectrum of light at a wavelength of $532 \mathrm{~nm}$. Compared to TURP, studies have demonstrated that the 80-W KTP laser has a superior safety profile $^{1}$ and encouraging postoperative results, including reduced catheterization time, hospital stay ${ }^{2}$ and transfusion requirement. $^{3}$

The evolution of PVP has occurred rapidly in an effort to improve efficacy and decrease operating time. The 120-W GreenLight HPS, launched in 2006, uses a lithium triborate crystal to produce a $532-\mathrm{nm}$ laser beam that is more

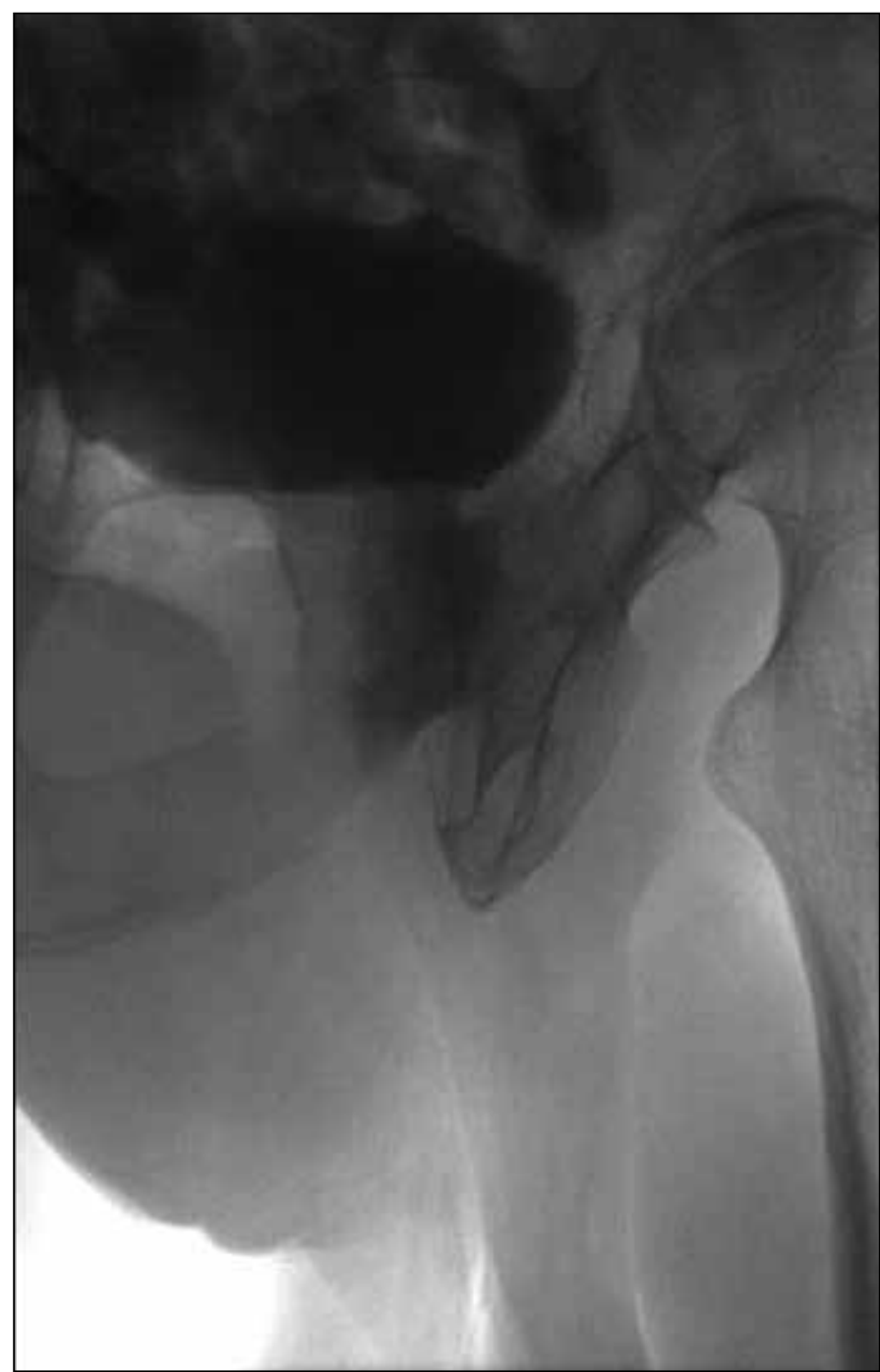

Fig. 2. Voiding cystourethrogram demonstrating extravasation of urine into the adductor compartment of the thigh.

powerful than the 80 -W KTP laser. ${ }^{4}$ This system enables more effective energy delivery per unit surface area, has a higher tissue ablation capacity ${ }^{5}$ and has a more efficient rate of prostatic tissue vaporization. Interestingly, it seems that the depth of tissue coagulation does not exceed 1 to $2 \mathrm{~mm}$ regardless of the power level used, suggesting that coagulation depth may be a function of wavelength and not higher power output. ${ }^{6}$ This finding implies that the GreenLight HPS laser should have a similar safety profile to the 80-W KTP laser; however, as Cleynenbreugel and colleagues observed in their review of the GreenLight HPS, this technology needs to be further studied to determine whether it will provide better outcomes while maintaining the same degree of patient safety as the $80-\mathrm{W}$ KTP laser. ${ }^{7}$

A valid critique of the GreenLight HPS laser is that randomized, prospective studies with long-term follow-up are scarce. In fact, only one mid-term randomized controlled 
trial on the efficacy and safety of the GreenLight HPS PVP compared to TURP has been produced to date. ${ }^{8}$ In this study, Al-Ansari and colleagues found that improvement in maximum flow rate, IPSS and PVR were comparable in both the PVP and TURP cohorts; however, perioperative complications, such as the need for transfusion, TURP syndrome and capsule perforation, were more common in those treated with TURP. Of note, more patients in the laser cohort suffered from dysuria postoperatively and more required a reoperation within the study period of 36 months.

With the U.S. Food and Drug Administration approval of the more powerful 180-W GreenLight XPS in late 2009, ${ }^{9}$ the incentive to pursue mid- and long-term data on the GreenLight HPS laser may be diminished. ${ }^{6}$ Already, the manufacture of this new product has reported the same safety profile, two times the speed of resection and better coagulation control than the GreenLight HPS. ${ }^{10}$ Unfortunately, high-quality clinical studies to support these claims are not currently available.

Ultimately, the safety and efficacy of the GreenLight laser depend on the operator. Adjustable power settings on these devices allow flexibility, if appropriate, to utilize higher power in situations where faster work is desired. If the high power setting is too close to the capsule, there may be an increased risk of serious complications, including prostatic capsular perforation and injury to adjacent structures. The case presented here is one of these serious but rare complications.

\section{Conclusion}

This case report demonstrates a number of important issues. Failure to recognize prostatic capsular perforation at the time of surgery can lead to serious complications. Also, poor continuity of care, due to patient and physician factors, increased morbidity and delayed diagnosis. Finally, GreenLight PVP is an evolving field in endourology, and it is important to evaluate safety and efficacy as experience with this technology expands.

Competing interests: None declared.

This paper has been peer-reviewed.

\section{References}

1. Boucher-Hayes DM, Van Appleton S, Bugeja P, et al. A randomized trial of photoselective vaporization of the prostate using the 80-W potassium-titanyl-phosphate laser vs transurethral prostatectomy, with a 1-year follow-up. BJU Int 2010;105:964-9. http://dx.doi.org/10.1111/i.1464-410X.2009.08961.x

2. Bachman A, Schurch L, Ruzet R, et al. Photoselective vaporization (PVP) versus transurethral resection of the prostate (TURP): a prospective bi-center study of peri-operative morbidity and early functional outcome. Eur Urol 2005;48:956-72. http://dx.doi.org/10.1016/i.eururo.2005.07.001

3. Ruszat $R$, Wyler $S$, Seitz $M$, et al. Comparison of potassium-itanyl-phosphate laser vaporization of the prostate and transurethral resection of the prostate: update of a prospective nonrandomized two-centre study. BJU Int 2008;102:1438-9.

4. Malek RS, Kang HW, Coad JE, et al. Green Light photoselective 120-watt 532-nm lithium triborate laser vaporization prostatectomy in living canines. J Endourol 2009;23:837-45. http://dx.doi.org/10.1089/ end.2008.0669

5. Heinrich E, Wendt-Nordahl $G$, Honeck $P$, et al. 120-W lithium triborate laser for photoselective vaporisation of prostate: comparison with 80-W potassium titanyl phosphate laser in an ex-vivo model. J Endourol 2010;24:75-9. http://dx.doi.org/10.1089/end.2009.0051

6. Reich 0. Greenlight: from potassium-titanyl-phosphate to lithium triborate or from good to better? Curr Opin Urol 2011;21:27-30. http://dx.doi.org/10.1097/MOU.0b013e3283410105

7. Cleynenbreugel BV, Srirangam SJ, Van Poppel H. High performance system Greenlight laser: indications and outcomes. Current Opin Urol 2009;19:33-7. http://dx.doi.org/10.1097/MOU.0b013e328317cab3

8. Al-Ansari A, Younes N, Sampige VP, et al. Greenlight HPS 120-W laser vaporization versus transurethral resection of the prostate for treatment of benign prostatic hyperplasia: a randomized clinical trial with midterm follow-up. Eur Urol 2010;58:349-55. http://dx.doi.org/10.1016/i.eururo.2010.05.026

9. FDA 2009. GreenlightTM XPS Laser System: 510(k) Premarket Notification. Retrieved May 10, 2011. www.accessdata.fda.gov/cdr__docs/pdf9/K092735.pdf. Accessed February 1, 2013.

10. American Medical Services 2010. Greenlight XPS. http://www.greenlighthps.com/XPS_intro.html. Accessed February 1, 2013.

Correspondence: Dr. David Harriman, 2775 Laurel St., Department of Urological Sciences, Vancouver, BC V5Z 1M9; davidiharriman@gmail.com 